\title{
PROPERTIES AND CHARACTERIZATIONS OF CONVEX FUNCTIONS ON TIME SCALES
}

\author{
Teodoro Lara, Nelson Merentes, Edgar Rosales, \\ Ambrosio Tineo
}

\begin{abstract}
In this research we deal with algebraic properties and characterizations of convex functions in the context of a time scale; this notion of convexity has been studied for some other authors but the setting of properties are establish here. Moreover, characterizations, a separation theorem and an inequality of Jensen type for this class of functions are shown as well.
\end{abstract}

\section{Introduction}

The theory of time scales was introduced by $\mathrm{S}$. Hilger in his $\mathrm{PhD}$ thesis [4. The calculus and applications of dynamic derivatives on time scales provide an unification and an extension of traditional differential and difference equations. In the same time, they can be seen as an unification of the discrete theory with the continuous theory. Also, it is a crucial tool in many computational and numerical applications. The applications of this calculus are substantial and they have received a lot of attention in the last years. The most important ones include the dynamic equations, which include both differential equations and difference equations [2]. At the present time, up to our knowledge, there are not too many results around on convex functions on a time scale besides the papers by C. Dinu [2, 3].

Received: 11.03.2017. Accepted: 02.12.201\%. Published online: 31.01.2018. (2010) Mathematics Subject Classification: 26A51, 32F17, 39B62.

Key words and phrases: time scales, convex functions, Jensen inequality. 
In this research we state and prove some properties on convex functions on time scales regarding algebraic properties and characterizations. On the real line $\mathbb{R}$ the concept of convex function is well known and may be seen in the available literature, for instance in [6, 9] and the references therein, we recall it here,

A function $f: I \rightarrow \mathbb{R}, I$ being a real interval, is called convex on $I$ if, for any $x, y \in I$ and $t \in[0,1]$,

$$
f(t x+(1-t) y) \leq t f(x)+(1-t) f(y) .
$$

The incoming notation and definitions are from [2] and [4]. A time scale (or measure chain) is any nonempty closed subset $\mathbb{T}$ of $\mathbb{R}$ (together with the topology of subspace of $\mathbb{R}$ ). Along this paper $\mathbb{T}$ will denote a time scale and, for any $I$ interval of $\mathbb{R}$ (open or closed), $I_{\mathbb{T}}=I \cap \mathbb{T}$ a time scale interval.

Definition 1.1. A function $f: I_{\mathbb{T}} \rightarrow \mathbb{R}$ is called convex on $I_{\mathbb{T}}$, if

$$
f(t x+(1-t) y) \leq t f(x)+(1-t) f(y)
$$

for all $x, y \in I_{\mathbb{T}}$ and all $t \in[0,1]$ provided that $t x+(1-t) y \in I_{\mathbb{T}}$. The function $f$ is strictly convex on $I_{\mathbb{T}}$ if the above inequality is strict for distinct $x, y \in I_{\mathbb{T}}$ and $t \in[0,1]$. The function $f$ is concave (respectively, strictly concave) on $I_{\mathbb{T}}$, if $-f$ is convex (respectively, strictly convex). A function that is both convex and concave on $I_{\mathbb{T}}$ is called affine on $I_{\mathbb{T}}$.

EXAMPLE 1.1. In [7] it was proved that the natural logarithm on a time scale, given by

$$
L_{\mathbb{T}}(t):=\int_{1}^{t} \frac{1}{\tau} \Delta \tau
$$

is a concave function on $\mathbb{T} \cap(0,+\infty)$. So, the function $f(t)=-L_{\mathbb{T}}(t)$ is convex on a time scale.

REMARK 1.2. It is clear that if a function $f: I \rightarrow \mathbb{R}$ is convex on $I$, then $f$ is convex on $I_{\mathbb{T}}$. That is, the usually convexity on intervals of $\mathbb{R}$ implies the convexity on a time scale; however, the converse is not true.

EXAmple 1.2. We consider $I=[0,2], \mathbb{T}=[0,1]$ and $f: I_{\mathbb{T}} \rightarrow \mathbb{R}$ given by

$$
f(x)=1-|x-1| .
$$


The function $\tilde{f}:[0,2] \rightarrow \mathbb{R}$ defined by $\tilde{f}(x)=|x|$ is convex, and it is such that $\left.\tilde{f}\right|_{I_{\mathbb{T}}}=f$. Therefore, $f$ is convex on $I_{\mathbb{T}}([2]$, Theorem 3.4), even when it is not convex on $I$.

We now set characterization of convex functions on a time scale similar to those given for convex functions and shown in [6, 9] among many others.

TheOREM 1.3. Let $f: I_{\mathbb{T}} \rightarrow \mathbb{R}$ be a function. The following statements are equivalent:

(1) $f$ is convex on $I_{\mathbb{T}}$.

(2) $f(x+t(y-x)) \leq f(x)+t(f(y)-f(x)), t \in(0,1), x, y \in I_{\mathbb{T}}$, provided $t x+(1-t) y \in I_{\mathbb{T}}$.

(3) $f(t x+s y) \leq t f(x)+s f(y), t, s \in(0,1), s+t=1, x, y \in I_{\mathbb{T}}$, provided $t x+s y \in I_{\mathbb{T}}$.

(4) If $x, y, z \in I_{\mathbb{T}}$ and $x<z<y$, then

$$
\operatorname{det}\left(\begin{array}{lll}
x & f(x) & 1 \\
y & f(y) & 1 \\
z & f(z) & 1
\end{array}\right) \geq 0 .
$$

(5) For $x, y, z \in I_{\mathbb{T}}$ with $x<z<y$,

$$
\frac{(y-z) f(x)+(y-x) f(y)+(x-z) f(z)}{(z-y)(z-x)(y-x)} \geq 0 .
$$

The set of convex functions on $I_{\mathbb{T}}$ will be denoted by $\mathcal{C}_{I_{\mathbb{T}}}$.

\section{The Results}

In this section we establish and prove our main results. First some simple algebraic properties of convex functions on a time scale similar to those given in [9] are stated. In almost all proofs we have to be aware that the corresponding convex combination of the type $t x+(1-t) y$, for $x, y \in I_{\mathbb{T}}$ and $t \in[0,1]$, is in $I_{\mathbb{T}}$, which make this stuff a little restrictive.

Proposition 2.1. If $f, g \in \mathcal{C}_{I_{\mathbb{T}}}$ and $\alpha \geq 0$, then $\alpha f$ and $f+g$ are in $\mathcal{C}_{I_{\mathbb{T}}}$ as well. 
Proof. For $x, y \in I_{\mathbb{T}}$ and any $t \in[0,1]$, provided $t x+(1-t) y \in I_{\mathbb{T}}$, by hypothesis

$$
\begin{aligned}
(f+g)(t x+(1-t) y) & \leq t f(x)+(1-t) f(y)+t g(x)+(1-t) g(y) \\
& =t(f+g)(x)+(1-t)(f+g)(y) .
\end{aligned}
$$

Proposition 2.2. Let $f \in \mathcal{C}_{I_{\mathbb{T}}}$ and $g \in \mathcal{C}_{J_{\mathbb{T}}}$ with range $(f) \subseteq J_{\mathbb{T}}$. If $g$ is increasing, then $g \circ f \in \mathcal{C}_{I_{\mathbb{T}}}$, where $\circ$ stands for composition of functions.

Proof. For $x, y \in I_{\mathbb{T}}$ and any $t \in[0,1]$ the only two things to worry about are that $t x+(1-t) y \in I_{\mathbb{T}}$ and at the same time $t f(x)+(1-t) f(y) \in J_{\mathbb{T}}$. The rest of the proof goes in the same fashion as the corresponding result for convex functions.

Proposition 2.3. Suppose $f, g \in \mathcal{C}_{I_{\mathbb{T}}}$ are nonnegative and increasing. If $h(x)=f(x) g(x), x \in J_{\mathbb{T}}$, then $h \in \mathcal{C}_{I_{\mathbb{T}}}$ and it is nonnegative and increasing as well. hence

Proof. Let $x, y \in I_{\mathbb{T}}$ with $x<y$, then $(f(x)-f(y))(g(y)-g(x)) \leq 0$,

$$
f(x) g(y)+f(y) g(x) \leq f(x) g(x)+f(y) g(y),
$$

this inequality will be used in the incoming set of inequalities. Now for any $t \in[0,1]$ and $t x+(1-t) y \in I_{\mathbb{T}}$,

$$
\begin{aligned}
f(t x+(1-t) y) g(t x+(1-t) y) \leq & t^{2} f(x) g(x)+t(1-t)[f(x) g(y)+f(y) g(x)] \\
& +(1-t)^{2} f(y) g(y) \\
\leq & t f(x) g(x)+(1-t) f(y) g(y) .
\end{aligned}
$$

THEOREM 2.4. Let $f_{\alpha} \in \mathcal{C}_{I_{\mathbb{T}}}$ be an arbitrary family, and $f(x)=\sup _{\alpha} f_{\alpha}(x)$, $x \in I_{\mathbb{T}}$. If $J_{\mathbb{T}}$ is the set given by $J_{\mathbb{T}}=\left\{x \in I_{\mathbb{T}}: f(x)<+\infty\right\} \neq \emptyset$, then $f \in \mathcal{C}_{J_{\mathbb{T}}}$.

Proof. For $x, y \in I_{\mathbb{T}}, t \in[0,1]$ and $t x+(1-t) y \in I_{\mathbb{T}}$,

$$
f(t x+(1-t) y) \leq t f_{\alpha}(x)+(1-t) f_{\alpha}(y) \leq t f(x)+(1-t) f(y) .
$$

THEOREM 2.5. If $f_{n} \in \mathcal{C}_{I_{\mathbb{T}}}$ is a sequence of functions converging to a finite limit function $f$ on $I_{\mathbb{T}}$, then $f \in \mathcal{C}_{I_{\mathbb{T}}}$. 
Proof. For $x, y \in I_{\mathbb{T}}, t \in[0,1]$ and $t x+(1-t) y \in I_{\mathbb{T}}$,

$$
\begin{aligned}
f(t x+(1-t) y) & \leq t \lim _{n \rightarrow+\infty} f_{n}(x)+(1-t) \lim _{n \rightarrow+\infty} f_{n}(y) \\
& =t f(x)+(1-t) f(y) .
\end{aligned}
$$

Proposition 2.6. Let $f \in \mathcal{C}_{I_{\mathbb{T}}}$. If $I_{\mathbb{T}}$ is compact, then $f$ is bounded from above.

Proof. Let $a:=\min I_{\mathbb{T}}, b:=\max I_{\mathbb{T}}$. If $x \in I_{\mathbb{T}}$, then there exists $t \in[0,1]$ such that $x=t a+(1-t) b$. Hence by hypothesis

$$
f(x)=f(t a+(1-t) b) \leq t f(a)+(1-t) f(b) \leq \max \{|f(a)|,|f(b)|\} .
$$

In [2] it is proved the following theorem, which connects the notions of convexity on a time scale and the usual convexity on $\mathbb{R}$.

Theorem 2.7. A function $f: \mathbb{T} \rightarrow \mathbb{R}$ is convex on $I_{\mathbb{T}}=I \cap \mathbb{T}$ if and only if there exists a convex function $\tilde{f}: I \rightarrow \mathbb{R}$ such that $\tilde{f}(x)=f(x)$ for all $x \in I_{\mathbb{T}}$.

Using this result, it is possible to prove the following characterization of convexity for functions on a time scale.

TheOREM 2.8. A function $f:(a, b)_{\mathbb{T}} \rightarrow \mathbb{R}$ is convex on a time scale if and only if there is an increasing function $g:(a, b) \rightarrow \mathbb{R}$ and a point $c \in(a, b)$ such that $f(x)-\int_{c}^{x} g(t) d t$ is constant for all $x \in(a, b)_{\mathbb{T}}$.

Proof. If $f$ is convex on $(a, b)_{\mathbb{T}}$ then, by Theorem 2.7, there exists a convex function $\tilde{f}:(a, b) \rightarrow \mathbb{R}$ such that

$$
\tilde{f}(x)=f(x) \text { for all } x \in(a, b)_{\mathbb{T}} .
$$

So, there is an increasing function $g:(a, b) \rightarrow \mathbb{R}$ and $c \in(a, b)$ such that for all $x \in(a, b), \tilde{f}(x)=\tilde{f}(c)+\int_{c}^{x} g(t) d t([9$, Theorem 12A]). Hence, from 2.1) it follows that for all $x \in(a, b)_{\mathbb{T}}$,

$$
f(x)-\int_{c}^{x} g(t) d t=k
$$

where $k$ is the constant $\tilde{f}(c)$. Notice that $k=f(c)$, if $c \in \mathbb{T}$. 
For the converse, we assume that for all $x \in(a, b)_{\mathbb{T}}, f(x)-\int_{c}^{x} g(t) d t$ is a constant, we say $k$ (since $c \in(a, b)$, the monotonicity of $g$ on $(a, b)$ implies that $\int_{c}^{x} g(t) d t$ exists for all $\left.x \in(a, b)\right)$. Define $\tilde{f}:(a, b) \rightarrow \mathbb{R}$ by

$$
\tilde{f}(x)=\int_{c}^{x} g(t) d t+k .
$$

Clearly, $\tilde{f}(c)=k$ and thus $\tilde{f}$ is convex on $(a, b)([9$, Theorem 12A]). Because $\tilde{f}(x)=f(x)$ for all $x \in(a, b)_{\mathbb{T}}$, Theorem 2.7 implies the convexity of $f$ on $(a, b)_{\mathbb{T}}$.

The next result is proved by using Theorem 2.7 too, and represents a discrete Jensen type inequality for functions defined on a time scale.

THEOREM 2.9. If $f: I_{\mathbb{T}} \rightarrow \mathbb{R}$ is a convex function (on a time scale) then, for all $n \in \mathbb{N}$,

$$
f\left(\sum_{i=1}^{n} t_{i} x_{i}\right) \leq \sum_{i=1}^{n} t_{i} f\left(x_{i}\right),
$$

for all $x_{1}, \ldots, x_{n} \in I_{\mathbb{T}}$ and $t_{1}, \ldots, t_{n}>0$ such that $\sum_{i=1}^{n} t_{i}=1$ and $\sum_{i=1}^{n} t_{i} x_{i} \in I_{\mathbb{T}}$.

Proof. If $f$ is convex on $I_{\mathbb{T}}$, then by Theorem 2.7 there exists a convex function $\tilde{f}: I \rightarrow \mathbb{R}$ such that $\tilde{f}(x)=f(x)$ for all $x \in I_{\mathbb{T}}$. From Jensen's inequality on a real interval ([9, Theorem $61 \mathrm{~A}])$,

$$
\tilde{f}\left(\sum_{i=1}^{n} t_{i} x_{i}\right) \leq \sum_{i=1}^{n} t_{i} \tilde{f}\left(x_{i}\right),
$$

for all $x_{1}, \ldots, x_{n} \in I$ and $t_{1}, \ldots, t_{n}>0$ with $\sum_{i=1}^{n} t_{i}=1$. The conclusion is obtained by restricting each $x_{i}$ belong to $I_{\mathbb{T}}$ with $\sum_{i=1}^{n} t_{i} x_{i} \in I_{\mathbb{T}}$.

In the following result we establish conditions that are necessary and sufficient to separate two functions (defined on a time scale) by a convex function (on the same time scale). The proof follows ideas from [8]. 
THEOREM 2.10. Let $f, g: I_{\mathbb{T}} \rightarrow \mathbb{R}$ be two functions defined on a time scale. Then, there exists a convex function $h: I_{\mathbb{T}} \rightarrow \mathbb{R}$ such that $f \leq h \leq g$ if and only if for any $n \in \mathbb{N}$,

$$
f\left(\sum_{i=1}^{n} t_{i} x_{i}\right) \leq \sum_{i=1}^{n} t_{i} g\left(x_{i}\right)
$$

for all $x_{1}, \ldots, x_{n} \in I_{\mathbb{T}}$ and $t_{1}, \ldots, t_{n}>0$ such that $\sum_{i=1}^{n} t_{i}=1$ and $\sum_{i=1}^{n} t_{i} x_{i} \in I_{\mathbb{T}}$.

Proof. $(\Rightarrow)$. By using the hypothesis and Theorem 2.9, we have that for any $n \in \mathbb{N}, x_{i} \in I_{\mathbb{T}}, t_{i}>0$ such that $\sum_{i=1}^{n} t_{i}=1$ and $\sum_{i=1}^{n} t_{i} x_{i} \in I_{\mathbb{T}}$,

$$
f\left(\sum_{i=1}^{n} t_{i} x_{i}\right) \leq h\left(\sum_{i=1}^{n} t_{i} x_{i}\right) \leq \sum_{i=1}^{n} t_{i} h\left(x_{i}\right) \leq \sum_{i=1}^{n} t_{i} g\left(x_{i}\right) .
$$

$(\Leftarrow)$. For each $x \in I_{\mathbb{T}}$, we consider the set

$$
C_{x}=\left\{\left(x_{1}, \ldots, x_{n}\right) \in\left(I_{\mathbb{T}}\right)^{n}: x=\sum_{i=1}^{n} t_{i} x_{i}, t_{i}>0, \sum_{i=1}^{n} t_{i}=1, n \in \mathbb{N}\right\},
$$

where $\left(I_{\mathbb{T}}\right)^{n}$ denotes the cartesian product $\underbrace{I_{\mathbb{T}} \times \cdots \times I_{\mathbb{T}}}_{n \text { factors }}$. Notice that $C_{x} \neq \emptyset$ since $(x, \ldots, x) \in C_{x}$. Therefore, the subset $G$ of $\mathbb{R}$ given by

$$
G=\left\{\sum_{i=1}^{n} t_{i} g\left(x_{i}\right):\left(x_{1}, \ldots, x_{n}\right) \in C_{x}\right\}
$$

is nonempty and, by (2.2), it is bounded from below.

Define the function $h: I_{\mathbb{T}} \rightarrow \mathbb{R}$ by

$$
h(x)=\inf G .
$$

Let $x$ be an arbitrary element in $I_{\mathbb{T}}$. By $(2.2), f(x)$ is a lower bound of $G$ and hence, $f(x) \leq h(x)$ on $I_{\mathbb{T}}$. Moreover, since $(x, \ldots, x) \in C_{x}$, we have that $g(x) \in G$ and thus, $h(x) \leq g(x)$ on $I_{\mathbb{T}}$. 
To see that $h$ is convex on $I_{\mathbb{T}}$, let $x, y \in I_{\mathbb{T}}$ and $\lambda \in[0,1]$ be such that $z:=\lambda x+(1-\lambda) y \in I_{\mathbb{T}}$. Then, by definition of $h$, we get for an arbitrary $\varepsilon>0$,

$$
h(x)+\varepsilon>\sum_{i=1}^{p} \alpha_{i} g\left(x_{i}\right) \text { for some }\left(x_{1}, \ldots, x_{p}\right) \in C_{x},
$$

and

$$
h(y)+\varepsilon>\sum_{j=1}^{q} \beta_{j} g\left(y_{j}\right) \quad \text { for some }\left(y_{1}, \ldots, y_{q}\right) \in C_{y} .
$$

Furthermore, since $x=\sum_{i=1}^{p} \alpha_{i} x_{i}$ and $y=\sum_{j=1}^{q} \beta_{j} y_{j}$, it follows that

$$
z=\sum_{i=1}^{p} \lambda \alpha_{i} x_{i}+\sum_{j=1}^{q}(1-\lambda) \beta_{j} y_{j}=\sum_{l=1}^{p+q} \gamma_{l} z_{l},
$$

where

$$
\gamma_{l}= \begin{cases}\lambda \alpha_{l}, & \text { for } l=1, \ldots, p, \\ (1-\lambda) \beta_{l-p}, & \text { for } l=p+1, \ldots, p+q,\end{cases}
$$

and

$$
z_{l}= \begin{cases}x_{l}, & \text { for } l=1, \ldots, p \\ y_{l-p}, & \text { for } l=p+1, \ldots, p+q .\end{cases}
$$

But $\sum_{l=1}^{p+q} \gamma_{l}=\sum_{l=1}^{p} \gamma_{l}+\sum_{l=p+1}^{p+q} \gamma_{l}=\sum_{i=1}^{p} \lambda \alpha_{i}+\sum_{j=1}^{q}(1-\lambda) \beta_{j}=1$. Thus $\left(z_{1}, \ldots, z_{p+q}\right) \in$ $C_{z}$ and hence, by definition of $h$ and using (2.3) and (2.4),

$$
\begin{aligned}
h(z) \leq \sum_{l=1}^{p+q} \gamma_{l} g\left(z_{l}\right) & =\sum_{l=1}^{p} \gamma_{l} g\left(z_{l}\right)+\sum_{l=p+1}^{p+q} \gamma_{l} g\left(z_{l}\right) \\
& =\sum_{i=1}^{p} \lambda \alpha_{i} g\left(x_{i}\right)+\sum_{j=1}^{q}(1-\lambda) \beta_{j} g\left(y_{j}\right) \\
& <\lambda[h(x)+\varepsilon]+(1-\lambda)[h(y)+\varepsilon] \\
& =\lambda h(x)+(1-\lambda) h(y)+\varepsilon .
\end{aligned}
$$

The convexity of $h$ on $I_{\mathbb{T}}$ follows by tending $\varepsilon \rightarrow 0^{+}$. 
Definition 2.11. Let $\varepsilon>0$ be a fixed real number. A function $f: I_{\mathbb{T}} \rightarrow \mathbb{R}$ is called $\varepsilon$-convex on a time scale if for any $n \in \mathbb{N}, x_{1}, \ldots, x_{n} \in I_{\mathbb{T}}, t_{1}, \ldots, t_{n}>0$ with $\sum_{i=1}^{n} t_{i}=1$ and $\sum_{i=1}^{n} t_{i} x_{i} \in I_{\mathbb{T}}$, we have

$$
f\left(\sum_{i=1}^{n} t_{i} x_{i}\right) \leq \sum_{i=1}^{n} t_{i} f\left(x_{i}\right)+\varepsilon .
$$

An immediate consequence of Theorem 2.10 is the following Hyers-Ulamtype stability result for convex functions on a time scale.

Corollary 2.12. If $f: I_{\mathbb{T}} \rightarrow \mathbb{R}$ is an $\varepsilon$-convex function on a time scale, then there exists a convex function $h: I_{\mathbb{T}} \rightarrow \mathbb{R}$ such that

$$
|f(x)-h(x)| \leq \varepsilon, x \in I_{\mathbb{T}} .
$$

Proof. We define the function $g: I_{\mathbb{T}} \rightarrow \mathbb{R}$ by $g(x)=f(x)+\varepsilon$ and apply Theorem 2.10.

\section{References}

[1] Bohner M., Peterson A., Dynamic equations on time scales. An introduction with applications, Birkhäuser, Boston, 2001.

[2] Dinu C., Convex functions on time scales, An. Univ. Craiova Ser. Mat. Inform. 35 (2008), 87-96.

[3] Dinu C., Hermite-Hadamard inequality on time scales, J. Inequal. Appl. 2008 (2008), Art. ID 287947, $24 \mathrm{pp}$.

[4] Hilger S., Analysis on measure chains-a unified approach to continuous and discrete calculus, Results Math. 18 (1990), 18-56.

[5] Kaymakçalan B., A survey of dynamic systems on measure chains, Funct. Differ. Equ. 6 (1999), 125-135.

[6] Merentes N., Rivas S., The development of the concept of convex function, XXVI Escuela Venezolana de Matemáticas, Venezuela, 2013 (in Spanish).

[7] Mozyrska D., Torres D.F.M., The natural logarithm on time scales, J. Dyn. Syst. Geom. Theor. 7 (2009), 41-48.

[8] Olbryś A., On separation by h-convex functions, Tatra Mt. Math. Publ. 62 (2015), 105-111.

[9] Roberts A.W., Varberg D.E., Convex functions, Academic Press, New York, 1973.

Departamento de Física y Matemáticas

Universidad de Los Andes

Trujillo

Venezuela

e-mail: tlara@ula.ve

e-mail: edgarr@ula.ve

e-mail: atemoya@ula.ve
ESCUELA DE MATEMÁTICAS

Universidad Central de Venezuela

Caracas

Venezuela

e-mail:nmerucv@gmail.com 